Original Article,

\title{
Risk of Unmasking on Schizophrenia Double Blind Clinical Trials Using Risperidone versus Comparator As A Result of Laboratory
} Analysis

\author{
Bogdan Viorel Barbu', Daniela Muresan², Cristian Gabos Grecu ${ }^{3}$, Prof. Iosif Gabos Grecu ${ }^{4}$ \\ ${ }^{1,2}$ University of Pharmacy and Medicine, George Emil Palade', Targu Mures Romania 38 Gheorghe \\ Marinescu Street, Targu Mures Romania \\ ${ }^{3,4}$ Emergency County Clinical Hospital Targu Mures $2^{\text {nd }}$ Psychiatry Department 38 Gheorghe Marinescu \\ Street, Targu Mures Romania \\ Email Address: Bogdan.barbu@ syneoshealth.com
}

\begin{abstract}
Background: Randomized double blind clinical trial is the most used and accepted method of clinical research involving human subjects. Clinical researches are performed under strict regulatory regulations including Helsinki Declaration and International Conference for Harmonization Good Clinical Practice (ICH - GCP) recommendations. As per ICH GCP principles safety and well being of clinical trials patients should prevail over research objectives and their health status should be monitored throughout the trial using objective and concise indicators such as but not limited to laboratory analysis.

This study aims to identify factors that might unblind risperidone treatment arms for patients enrolled in randomized double blind schizophrenia clinical trials using risperidone versus comparator, as a result of reviewing safety laboratory analysis.

Methods: This study included 134 controlled schizophrenia patients who received treatment with risperidone versus antipsychotic agents with lower dopamine antagonist potency. Prolactine values were determined using imunoenzimatic and colorimetric method (ELISA) for cantitative determination of prolactine from serum or plasma.

Results: $84 \%$ percent of the patients treated with risperidone showed elevated levels for prolactine above laboratory reference ranges defined as: Men ( $1.8-17.0 \mathrm{ng} / \mathrm{ml}$ ) ; Women ( fertile $1.2-19.5 \mathrm{ng} / \mathrm{ml}$, menopause $1.5-18.5 \mathrm{ng} / \mathrm{ml}$ )

Conclusions: High elevated values of prolactin were determined for risperidone treated patients which might disclose the patient treatment arm to blinded investigator and jeopardize the research. As lab results are an essential safety factor of clinical trial those cannot be overruled and establishing Independent Data Monitoring Committee to oversee patients' data and warn the investigator is recommended.
\end{abstract}

Keywords: Schizophrenia; clinical trials; prolactine; laboratory assessments.

\section{Introduction:}

Good Clinical Practices (GCP) constitutes an international ethical and scientific quality standard for designing, conducting recording and reporting clinical trials that involve the participation of Human subjects. Therefore compliance with it's standard provides public assurance that the rights, safety and well being of trial subjects are protected with the principles that have their origin in the Declaration of Helsinki, and that the clinical trials are credible (World Health Organization).

The main objectiveof ICH (international Conference of Harmonization ) GCP Guideline is providing an unified standard for the European Union (EU), Japan, and the United States to facilitate the mutual acceptance of clinical trials information by the regulatory authorities in these Jurisdictions( 1 )

Clinical trials are the type of research which studies new treatments and tests to evaluates their 


\section{Bogdan Viorel Barbu et.al / Risk of Unmasking On Schizophrenia Double Blind Clinical Trials Using Risperidone versus Comparator As A Result of Laboratory Analysis}

effects on humans health outcome. The participants volunteer to take part in such clinical trials in order to test medical interventions including medicines, cells and other types of biological products, surgical procedures, radiological procedures, devices, behavioral treatments and preventive care (2)

The designof clinical trial is a very important aspect of interventional trials which serves to optimize, facilitate, and economize the conduct of the clinical trial, as the purpose of the clinical research is to assess the efficacy, safety, and risk benefit ratio for a certain treatment.

Clinical trials follow a hierarchy in terms of the quality of evidence that they can provide. Clinical trials double blind randomized placebo control (RDBPC) studies are considered the "gold standard" of epidemiologic studies. (3) In the context of a clinical researh, double-blind means that neither the subject nor the members of investigational team are aware who is getting a placebo and who is receiving the treatment. Because subjects don't know what kind of treatment they are receiving, their belief about what will happen doesn't affect the results. Because the members of investigational teamare not aware either, they can't hint to patients about what kind of treatment they receive, and they also won't affect the results through their own biased expectations about what the results will be.

Bias refers to a deviation from the truth in the process of collection, analysis, interpretation, or publication of results, leading to false or wrong conclusions. Poor or insufficient blinding of a clinical trial may conduct to bias and thismay lead to a result in an inflated effect size and may increase the risk of type I error. In this regard even a small error in blinding may determine a statistically significant result without any real difference between the study groups. (4) Keeping both the physicians and the patients blinded to treatment allows a double-blinded study to limit the capacity of physicians to treat the study groups differently. The double-blinded clinical research minimizes the risk of various types of biases, such as observer bias or confirmation bias, which may jeopardize the results of the clinical trial. (5-6) It also prevent to avoid a disproportionately large placebo effect in the subjects involved in the study. (7)

Schizophrenia is a severe mental illness characterized by psychosis and mood and cognitive disorder. The clinical signs and symptoms of schizophrenia are very complex and display different patterns which vary widely from patient to patient. The manifestation of positive symptoms is not constant and tends to fluctuate over time, whereas negative symptoms and cognitive impairment are usually more persistent with less fluctuation over time. The pathophysiology of schizophrenia remains unclear; however, treatment via modulation of the dopamine and serotonin systems has received attention. In 1994, Janssen Pharmaceutical developed risperidone, which combined potent serotonin Type 2A (5HT2A) and dopamine Type 2 (D2) blockade. Risperidone is now a well established atypical antipsychotic for treatment of Schizophrenia (8).

The potential toxicity associated with the utilization of risperidone is known due to its wide use in human therapy. The most common adverse events reported in literature are dose-dependent andare a result of the pharmacological activity of risperidone. Also, they are mainly the result of Prolactin-mediated levels (9). Adverse events and/or laboratory abnormalities identified in the developed protocol of the clinical trial as critical to safety evaluations need to be reported to the Sponsor (defined as the organization who developed the investigative product) according to the reporting requirements and within the time periods specified in the protocol

The nature and purpose of laboratory work conducted as part of a clinical trial is extremely broad. Laboratories perform a large range of activities which provide data that is used to monitor trial subject safety, assess pharmacokinetic parameters and to measure end points.

Appropriate procedures need to be implemented to ensure effective and timely communication with the sponsor or their representative, regarding any serious deviations from the work instruction, clinical trial protocol or contract/agreement. Regulate reporting will ensure that the sponsor or their representative are able to determine the significance and impact of the deviation on the safety and well being of the trial subjects and on the integrity and reliability of the trial data. (10)

\section{Materials and methods:}

This study aims to identify factors that might unblind risperidone treatment arms for patients enrolled in randomized double blind 


\section{Bogdan Viorel Barbu et.al / Risk of Unmasking On Schizophrenia Double Blind Clinical Trials Using Risperidone versus Comparator As A Result of Laboratory Analysis}

schizophrenia clinical trials using risperidone versus comparator, as a result of reviewing safety laboratory analysis.

Methods: This study included 134 controlled schizophrenia patients who received treatment with risperidone versus antipsychotic agents with lower dopamine antagonist potency. The subject were selected in a ratio $1: 1$ for treatment with risperidone and treatment with other antipsychotic. Although there were no direct interference on the subjects treatments the research aimed to the subject stable to schizophrenia symptoms. For this they were evaluated using PANSS (Positive and Negative Symptoms in Schizophrenia) probe and they need to have a result between 65 and 100 points. Nevertheless an informed consent form was used to obtain subject agreement to use and interpret their lab reports results on prolactine levels. Prolactine values were determined using imunoenzimatic and colorimetric method (ELISA) for cantitative determination of prolactine from serum or plasma.

Statistical analysis: $\mathrm{T}$ Student test with pairedsamples.

\section{Results:}

$84 \%$ percent of the patients treated with risperidone showed elevated levels for prolactine above laboratory reference ranges defined as: Men ( $1.8-17.0 \mathrm{ng} / \mathrm{ml}$ ) ; Women ( fertile $1.2-19.5$ $\mathrm{ng} / \mathrm{ml}$, menopause $1.5-18.5 \mathrm{ng} / \mathrm{ml}$ )

\section{Discussions:}

According with principles of ICH GCP ( International Conference for Harmonization Good Clinical practices ), clinical trials should be conducted in accordance with the ethical Principles that gave their origin in the Declaration of Helsinki and that are consistent with GCP and the applicable regulatory requirements(s).

The rights safety, and well being of the subjects enrolled in clinical research are the most important considerations and should prevail over interest of science and society and all clinical research information should be recorded, handled, and stored in a way that allows its accurate reporting, interpretation and verification.

As per same principles the investigator responsibilities during and following a subject participation in a trial, the investigator/ institution should ensure that adequate medical care is provided to a subject for any adverse events, including clinically significant laboratory values related to the trial. The investigator/ institution should inform a subject when medical care is needed for intercurrent illness (es), of which the investigator became aware.

Therefore in situation of double blinded clinical trials involving risperidone as one or more treatment arms the clinical lab results is a main tool in assessing the patient safety, well being and potential adverse events throughout clinical trial by the investigative team. On the same time elevated prolactin values will disclose to investigational team the assignment of the patient on the study treatment arms jeopardizing the objectives of the research. In order to mitigate this potential contradictory situation in which the investigative teamif will have access to crucial data for patient safety will determine patient assignment on treatment arm and will lead trial results toa bias, the most obvious solution might be blinding the lab results to the investigative team. However, in order to respect GCP principles those results will be fully disclosed to an independent data monitoring committee who will assess patient laboratory results and will decide if at a certain moment a patient will be at risk. As soon as prolactin lab results will exceed with a certain degree the normal lab results for prolactin and will pose a risk for further participation of patient into the clinical trial the committee will notify the investigational team with strong recommendation of withdrawing the patient from clinical trial.

Once the patient is withdrawn from the trial all his/her laboratory values will be disclosed to investigational team without the bias risk for clinical research, and the best standard of care will be provided to the patient.

\section{Conclusions:}

High elevated values of prolactin were determined for risperidone treated patients within double blind clinical trials which might disclose the patient treatment arm to blinded investigator and jeopardize the research. As lab results are an essential safety factor of clinical trials those cannot be overruled and establishing an Independent Data Monitoring Committee to oversee patient's data and warn the investigator is highly recommended. 


\section{Bogdan Viorel Barbu et.al / Risk of Unmasking On Schizophrenia Double Blind Clinical Trials Using Risperidone versus Comparator As A Result of Laboratory Analysis}

The authors have no competing interests in this research.

\section{Bibliography:}

[1] New revised printing of revisd April 1, 2003 Code of federal Regulations and ICH Guidelines (Good Clinical Practices and Clinical Safety Data Management) plus The European Union Clinical trials Directive.

[2] Brijesh Nair - Indian Dermatology Journal 2019 Mar-Apr; 10(2): 193-201.

[3] Shobha Misra-Indian J Sex Transm Dis AIDS. 2012 Jul-Dec; 33(2): 131-134.

[4] Chen L, Su Y, Quan L, Zhang Y, Du L. Clinical Trials Focusing on Drug Control and Prevention of Ventilator-Associated Pneumonia: A Comprehensive Analysis of Trials Registered on ClinicalTrials.gov. Front Pharmacol. 2018;9:1574.

[5] Platt RW, Platt R, Brown JS, Henry DA, Klungel $\mathrm{OH}$, Suissa S. How pharmacoepidemiology networks can manage distributed analyses to improve replicability and transparency and minimize bias. Pharmacoepidemiol Drug Saf. 2019 Jan 15;
[6] Clifton L, Clifton DA. How to maintain the maximal level of blinding in randomisation for a placebo-controlled drug trial. Contemp Clin Trials Commun. 2019 Jun;14:100356.

[7] Probst P, Zaschke S, Heger P, Harnoss JC, Hüttner FJ, Mihaljevic AL, Knebel $\mathrm{P}$, Diener MK. Evidence-based recommendations for blinding in surgical trials. Langenbecks Arch Surg. 2019 May;404(3):273-284.

[8] Chengappa K.N.R. Sheth S., Brar S.J., et al, A clinical audit of the first 142 patients who received risperidone at a state psychiatric hospital. J Clin Psychiat 1999; 60:373-8.

[9] Conley R.R., Kelly D.L., Management of treatment resistance in schizophrenia. Biol Psyhiat 2001; 50:898-911

[10] $28 \quad$ February 2012 EMA/INS/GCP/532137/2010 GCP Inspectors Working Group Reflection paper for laboratories that perform the analysis or evaluation of clinical trial samples. European Medical Agency. 